\title{
FIGO Stage IC1
}

National Cancer Institute

\section{Source}

National Cancer Institute. FIGO Stage IC1. NCI Thesaurus. Code C128099.

A FIGO stage term that applies to ovarian cancer, fallopian tube cancer, and primary peritoneal cancer. It refers to cancer with surgical spill. 